\title{
Preparation of Cylindrical Janus Particles Using a Stirring Method
}

\author{
Miku Onishi, Yuya Tsujishita, Wei Li, Toyoko Suzuki, and Hideto Minami*
}

Cite This: ACS Omega 2020, 5, 33047-33052

Read Online

ABSTRACT: We previously discovered a novel method for the preparation of polymer particles that have a cylindrical shape. Polystyrene (PS) or poly methyl methacrylate (PMMA) spherical particles were deformed into a cylindrical shape by stirring with a magnetic stirrer in a polyvinylpyrrolidone (PVP) aqueous solution. In this study, cylindrical "Janus" particles consisting of PS and PMMA were prepared by this stirring method. In the case of spherical Janus particles, cylindrical particles were obtained after stirring; however, the direction of the interface between the PS and PMMA phases was random. However, in the case of snowman-like Janus particles, cylindrical Janus particles with the interface at the center of the long axis were successfully prepared. This indicated that the extension direction can be controlled owing to the
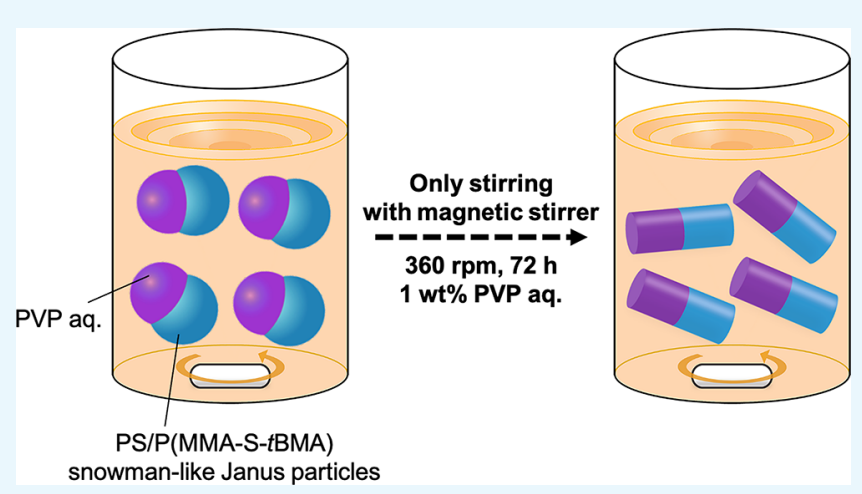
anisotropic shape and supported the proposed deformation mechanism of the cylindrical particles. Moreover, amphiphilic cylindrical Janus particles were also successfully prepared by hydrolysis of only one phase to introduce carboxy groups.

\section{INTRODUCTION}

Typically, polymer particles prepared by heterogeneous polymerization tend to become spherical in shape to minimize the interfacial free energy between the particles and medium. However, nonspherical polymer particles have unique properties, such as mechanical, rheological, and optical properties, and they are applied in paint as antisagging agents and in cosmetics. $^{1,2}$ Recently, we reported that polystyrene (PS) or polymethylmethacrylate (PMMA) spherical particles could be transformed into a cylindrical shape by stirring in a polyvinylpyrrolidone (PVP) aqueous solution. ${ }^{3}$ We proposed a mechanism of particle deformation for the stirring method as follows: the particles were plasticized by the PVP aqueous solution and then stretched by applying shear stress from the medium by stirring with a magnetic stirrer. This method provides a facile and highly efficient approach for preparing cylindrical particles, which is distinguishable from the ellipsoidal shape obtained by other previous methods. Zou et al. recently reported the preparation of cylinder-like PS-silica composite particles using the same method. ${ }^{4}$

Janus particles are composite particles ${ }^{5}$ that have two different surfaces on each half. There are many reports about preparation methods for Janus particles, such as Pickering emulsion, ${ }^{6,7}$ toposelective surface modification, ${ }^{8,9}$ phase separation method by seeded polymerization, ${ }^{1,10}$ and microfluidic method. ${ }^{11,12}$ Nonspherical Janus particles can be also synthesized by using the microfluidic technique ${ }^{13}$ and selfassembly of block copolymers, ${ }^{14}$ besides the molding method. These particles can be used as particulate surfactants ${ }^{15}$ and as building blocks for a colloidal structure. ${ }^{16}$ For example, in the case of Janus particles with hydrophobic and hydrophilic surfaces, micelle-like particles were formed in aqueous solution. ${ }^{17}$ We succeeded in arranging a one-dimensional colloidal structure of Janus particles that includes different stabilizers on each side by utilizing hydrogen-bonding interactions. ${ }^{18}$ Moreover, Janus particles that have a nonspherical shape are expected to have different assembly behaviors from spherical particles when used as building blocks for colloidal structures. ${ }^{19}$ Matchstick-shaped Janus particles produce a unique structure that cannot be produced by spherical Janus particles. ${ }^{20}$ Lee et al. reported the control of the self-assembly of Janus particles that have a cylindrical shape by changing the polarity of the medium. ${ }^{21}$ These cylindrical Janus particles were prepared by a sequential micromolding method, which still involves a complicated procedure.

In this study, we attempted to prepare Janus particles that have a cylindrical shape consisting of PS and PMMA by the stirring method. Using this method, we expected to easily synthesize such Janus particles having a cylindrical shape. Additionally, we demonstrated the preparation of amphiphilic cylindrical Janus particles.

Received: September 16, 2020

Accepted: December 7, 2020

Published: December 17, 2020 

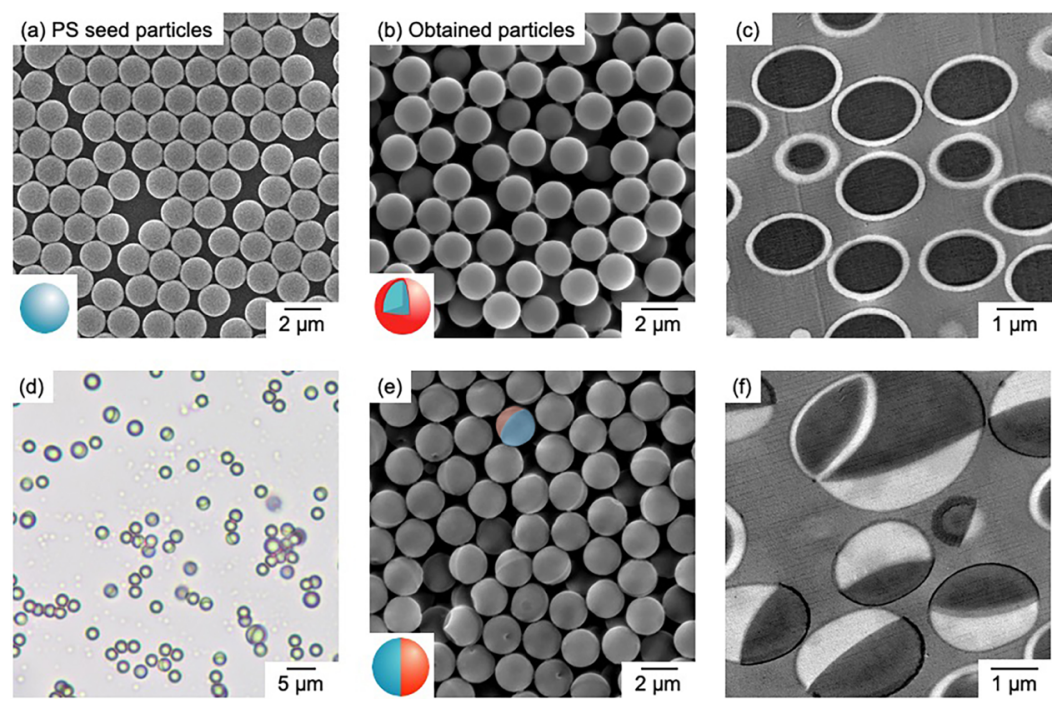

Figure 1. SEM image of PS seed particles prepared by dispersion polymerization (a). SEM image (b) and TEM image of ultrathin cross sections (c) of the obtained particles prepared by seeded dispersion polymerization of MMA. Optical micrograph (d), SEM image (e), and TEM image of ultrathin cross sections (f) of PS/PMMA composite particles after the SARM process in the presence of SDS. Particles of $(\mathrm{c}, \mathrm{f})$ were stained with $\mathrm{RuO}_{4}$ vapor.

\section{RESULTS AND DISCUSSION}

First, $1.78 \mu \mathrm{m}$ monodisperse PS particles were obtained via dispersion polymerization (Figure 1a). The seeded dispersion polymerization of methyl methacrylate (MMA) was carried out using these PS particles as the seeds, resulting in $2.03 \mu \mathrm{m}$ particles. The diameters of the obtained particles were larger than those of the PS seed particles (Figure 1b), suggesting that composite particles were successfully prepared. From the transmission electron microscopy (TEM) observation of the ultrathin cross sections of the obtained particles stained with $\mathrm{RuO}_{4}$, which preferentially stains the PS phase, clearly, the obtained particles had a core-shell structure that consisted of a PS core and a PMMA shell (Figure 1c).

To change the morphology from core-shell to Janus, the solvent-absorbing/releasing method (SARM) process in the presence of emulsifiers on the core-shell composite particles was carried out in which the interfacial tension values between the polymer phases and the medium decreased. Figure 1e shows the scanning electron microscopy (SEM) image of the composite particles after the SARM process in the presence of sodium dodecyl sulfate (SDS). After the SARM process, the obtained particles were spherical, and the size was not changed. However, the phase boundary was likely observed at the center of the particles according to the optical micrograph (Figure 1d). To identify the phase boundary of the Janus particles, one particle in SEM was colored (Figure 1e). According to the TEM image of the ultrathin cross sections of the composite particles (Figure 1f), the inside of the particles changed to a Janus structure. Although a composite particle having irregular morphology was observed (upper in Figure 1f) due to coagulation during the preparation of ultrathin cross sections, such a particle was rarely observed.

To deform the spherical Janus particles into a cylindrical shape, they were stirred in a 1 wt \% PVP aqueous solution (solid content: 1 wt \%) using a magnetic stirrer (360 rpm) at room temperature, which were the optimal conditions for deformation in our previous paper. ${ }^{3}$ High stirring speed and high temperature would induce formation of irregularly shaped particles. However, most of the particles were not deformed and remained as spherical shapes even after stirring for $72 \mathrm{~h}$ under the optimal conditions. The result was based on the difference in the degree of plasticization by PVP between the PS and PMMA phases, although the reason was not clear. To confirm this, PS and PMMA homopolymer particles were separately stirred in a PVP aqueous solution under the same conditions. After stirring for $72 \mathrm{~h}$, approximately $80 \%$ of the PS particles deformed into a cylindrical shape, whereas only approximately $50 \%$ of the PMMA particles deformed (only the deformed particles with the aspect ratio of more than 1.1 were counted), and the length of the cylindrical shape was shorter than that of the PS cylindrical particles (Figure S1). Moreover, PS and PMMA homopolymer particles were stirred together in a PVP and coumarine 6 (fluorescence dye) aqueous solution for $72 \mathrm{~h}$, in which coumarine 6 preferentially adsorbed to the PMMA particles. Both polymer particles were stirred together in a PVP aqueous solution, and the deformation degree of the PS particles (non-fluorescence) was larger than that of the PMMA particles (fluorescence) (Figure 2), indicating that the PS phase should be more easily plasticized than the PMMA phase. Thus, to approach the degree of plasticization between PS and PMMA phases in composite particles, $10 \mathrm{wt} \%$ styrene
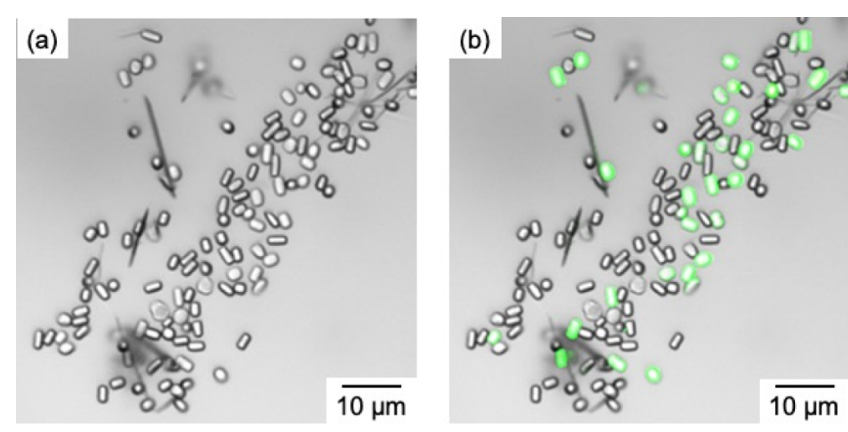

Figure 2. Optical micrograph (a) and confocal laser scanning microscopy image (b) of PS and PMMA particles dispersed in aq coumarine 6 after stirring in aq PVP for $72 \mathrm{~h}$. PMMA particles produced green fluorescence. 
based on MMA was polymerized with MMA in the seeded polymerization. After seeded dispersion copolymerization, the core-shell composite particles [2.09 $\mu \mathrm{m}$ (using $1.78 \mu \mathrm{m}$ PS seed particles)] were also successfully prepared (Figure 3a,b). After the SARM process, PS/poly(MMA-styrene) [PS/ $\mathrm{P}(\mathrm{MMA}-\mathrm{S})]$ Janus particles were also obtained in the same way as the PS/PMMA particles (Figure 3c,d).
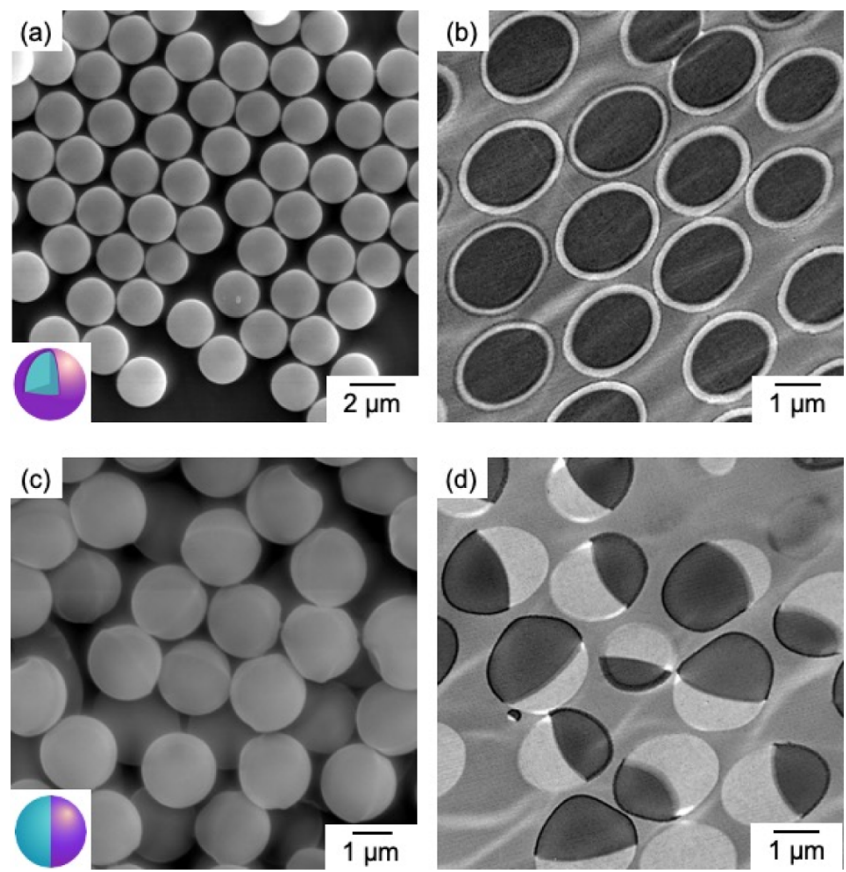

Figure 3. SEM image (a) and TEM image of the ultrathin cross sections (b) of the obtained particles prepared by seeded dispersion polymerization of MMA and styrene. SEM image (c) and TEM image of the ultrathin cross sections (d) of the PS/P(MMA-S) composite particles after the SARM in the presence of SDS. Particles of $(b, d)$ were stained in the PS phase with $\mathrm{RuO}_{4}$ vapor.

Figure 4 shows the optical micrograph and SEM image of the PS/P(MMA-S) Janus particles after stirring in a PVP aqueous solution. Unlike the case of PS/PMMA Janus particles, more than half of the particles were successfully deformed into a cylindrical shape, and the average aspect ratio of the cylindrical particles was around 1.5; distribution is shown in Figure S2. However, after removal of the PMMA phase of these particles with acetic acid (a good solvent for PMMA but not for PS), the position and direction of the interface between the PS and PMMA phases were random (Figure $4 c$ ). In the case of the spherical particles, the elongation direction was random in the flow during stirring because of the isotropy of the particles. To obtain cylindrical Janus particles that had a boundary with the center of the cylindrical particles, anisotropic snowman-like Janus particles were used.

The preparation of snowman-like Janus particles has been reported, in which the SARM process was carried out using nonionic Emulgen 911 instead of SDS. ${ }^{22}$ Figure 5a,b shows the optical micrograph and SEM image of the composite particles after the SARM process in the presence of Emulgen 911. The shape of the obtained particles changed from spherical to snowman-like. According to the TEM image of the ultrathin cross sections of these particles (Figure $5 c$ ), the insides of the particle were also changed to Janus structures (snowman-like shapes). The aspect ratio of snowman-like particles was about 1.2 , which should be enough to align with the shear direction.

Figure 6a,b shows the optical micrograph and SEM image of the snowman-like Janus particles after stirring in a PVP aqueous solution. Around $70 \%$ of the particles were also deformed into a cylindrical shape, although some particles had an ellipsoid shape. The aspect ratio statistics of the deformed particles are also shown in Figure S3. When the PMMA phase of these particles was extracted with acetic acid, the interface between the polymer phases was uniformly positioned perpendicular to the center of the long axis of all particles (Figure 6c). This result supports the deformation mechanism for the stirring method.

Additionally, to prepare amphiphilic cylindrical Janus particles, the PMMA phase was hydrolyzed to introduce carboxy groups. In the seeded dispersion polymerization, $10 \mathrm{wt}$ $\%$ tert-butyl methactylate ( $t \mathrm{BMA})$ based on MMA and styrene was polymerized with MMA and styrene because PtBMA is susceptible to hydrolysis compared with PMMA. After the seeded dispersion polymerization, PS/poly(MMA-styrenetBMA) [PS/P(MMA-S- $t$ BMA)] spherical composite particles were also obtained (Figure S4), and then, snowman-like Janus particles were prepared using the SARM process in the same manner (Figure 7a). PS/P(MMA-S-tBMA) cylindrical Janus particles were also prepared via the stirring method (Figure $7 \mathrm{~b}$ ) in PVP aqueous medium. To introduce the carboxy groups on half of the cylindrical Janus particles, the tert-butyl group of the PMMA phase was hydrolyzed using a trifluoroacetic acid (TFA) aqueous solution. The shapes of the particles did not change after the TFA treatment (Figure 7c). To confirm the presence of a carboxy group, Fourier-transform infrared spectroscopy (FT-IR) measurements were carried out; however, the characteristic peaks were not observed owing to their small intensity (carboxy groups were only on the surface of the PMMA phase). However, a Pickering emulsion was
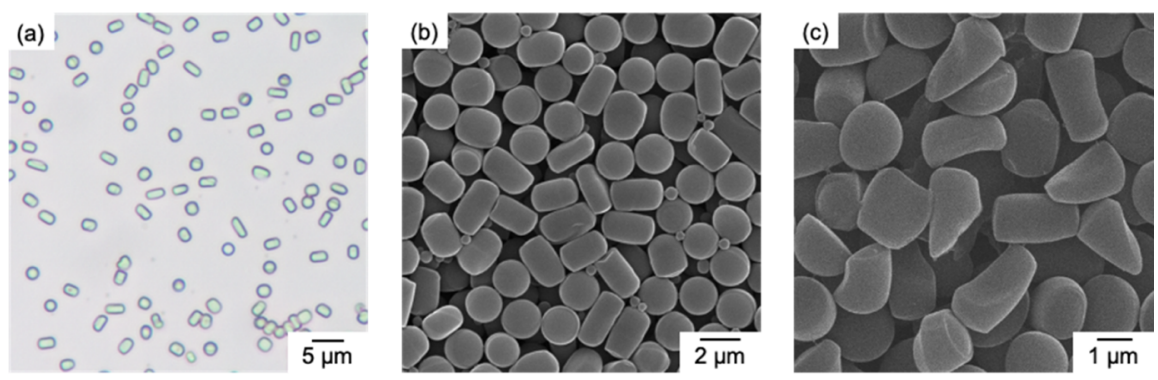

Figure 4. Optical micrograph (a) and SEM image (b,c) of PS/P(MMA-S) cylindrical Janus particles after stirring in 1 wt \% PVP aqueous solution for $72 \mathrm{~h}$. The particles shown in (c) are the removed PMMA phase (P(MMA-S) portion) particles with acetic acid. 

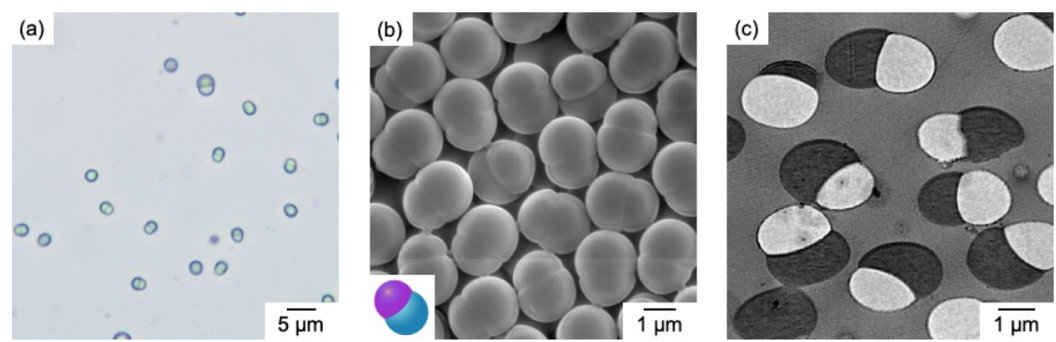

Figure 5. Optical micrograph (a) and SEM (b) and TEM images of the ultrathin cross sections (c) of the PS/P(MMA-S) composite particles after the SARM process in the presence of Emulgen 911. The particles shown in (c) were stained in the PS phase with $\mathrm{RuO}_{4}$ vapor.
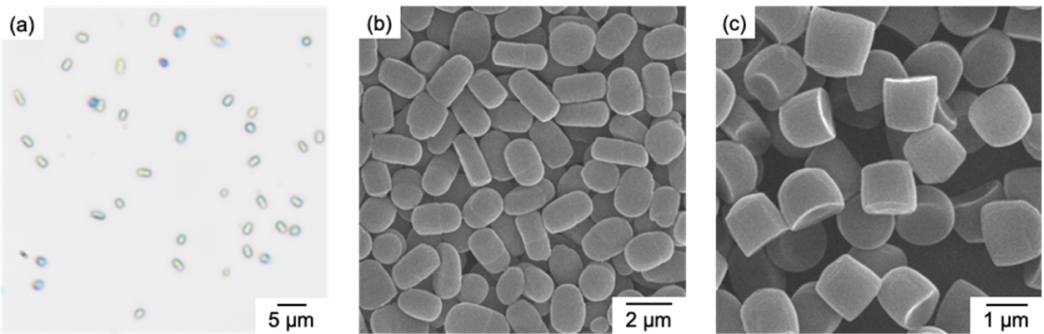

Figure 6. Optical micrograph (a) and SEM images (b,c) of PS/P(MMA-S) cylindrical Janus particles prepared by stirring the PS/P(MMA-S) snowman-like Janus particles in $1 \mathrm{wt} \%$ PVP aqueous solution for $72 \mathrm{~h}$. The particles shown in (c) were the removed PMMA phase (P(MMA-S) portion) particles with acetic acid.
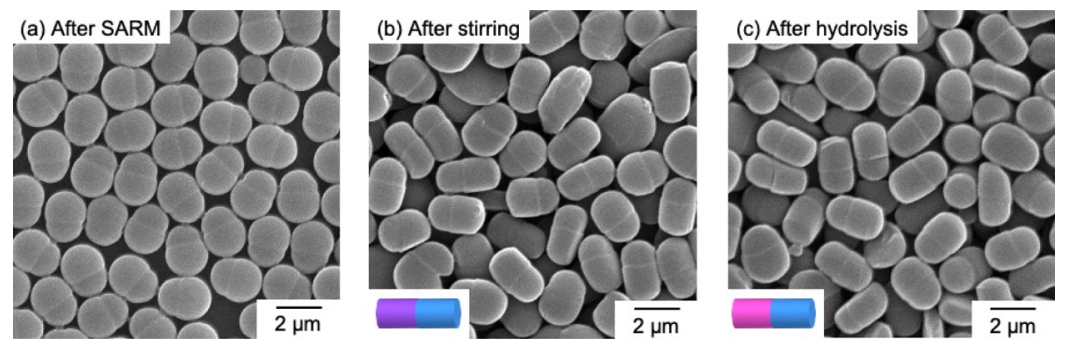

Figure 7. SEM images of the PS/P(MMA-S-tBMA) composite particles after the SARM in the presence of Emulgen 911 (a), after stirring in 1 wt \% PVP aqueous solution for $72 \mathrm{~h}(\mathrm{~b})$ and after hydrolysis by $5.6 \mathrm{wt} \%$ TFA aqueous solution (c).

prepared using these particles as a particulate surfactant, and there was a large difference in the adsorption behavior of the particles at the oil droplet interface. Regarding the particles before hydrolysis, the long axis of the adsorbed particle became parallel to the interface of the oil droplet (Figure $8 \mathrm{a}$ ) owing to the increased adsorption area, ${ }^{23}$ which also indicated that both phases of the Janus particles had the same hydrophobicity. However, in the case of the hydrolyzed Janus cylindrical particles, the cylindrical particles adsorbed vertically to the
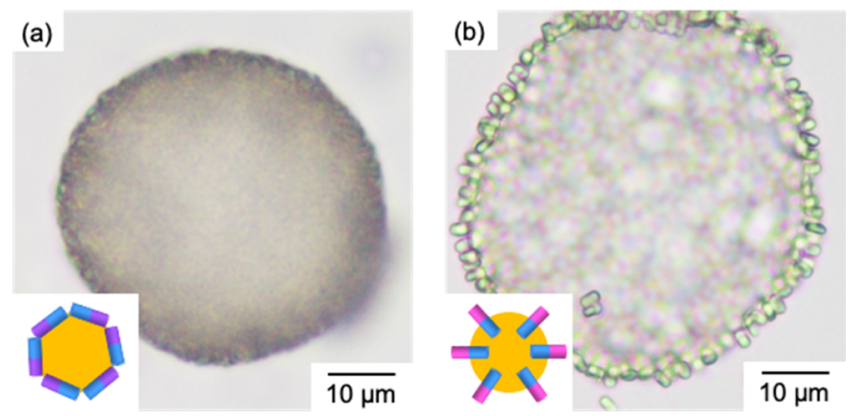

Figure 8. Optical micrographs of the Pickering emulsion using PS/ $\mathrm{P}$ (MMA-S-tBMA) cylindrical Janus particles before $(\mathrm{a}, \mathrm{b})$ and after hydrolysis. interface (Figure 8b). By through-focus observation (Figure S5), the adsorption behaviors can be more clearly observed. This result strongly suggests that the carboxy groups were introduced to the surface of one side of the Janus particles, that is, amphiphilic cylindrical Janus particles were successfully prepared. In the case of this adsorption method, the emulsion is expected to be more stable because the adsorption energy of the particles at the oil-water interface became large. We also tried to observe the adsorption behavior of Janus particles on oil droplet with SEM by replacing the oil phase from octane to hexadecane, for which the melting point is above room temperature. However, the behavior could not be observed because the hexadecane melted and the adsorbed morphology of the cylindrical particles had collapsed during the observation with the SEM.

\section{CONCLUSIONS}

We produced cylindrical Janus particles by stirring snowmanlike Janus particles prepared by seeded dispersion polymerization and the SARM process. Control of the elongation direction of the particles by using anisotropic particles was important for preparation of the cylindrical Janus particles that had a boundary with the center of the cylindrical particles. These results also strongly supported the elongation mechanism of the particles during stirring. Additionally, these 
amphiphilic cylindrical Janus particles are expected to be used for the preparation of colloidal structures via self-assembly.

\section{EXPERIMENTAL PROCEDURE}

Materials. Styrene, MMA, and tBMA (Nacalai Tesque, Inc., Kyoto, Japan) were purified via distillation under reduced pressure in a $\mathrm{N}_{2}$ atmosphere. Reagent-grade 2,2'-azobis(isobutyronitrile) (Wako Pure Chemical Industries, Osaka, Japan) and 2,2'-azobis-(2,4-dimethyl valeronitrile) (V-65, Wako Pure Chemical Industries, Osaka, Japan) were purified by recrystallization in methanol. Reagent-grade methanol, ethanol, toluene, octane, $\mathrm{NaOH}$, TFA, PVP (K-30; weightaverage molecular weight $=4.0 \times 10^{4}$, and K-15; $M_{\mathrm{w}}=1.0 \times$ $10^{4}$, Nacalai Tesque, Inc., Kyoto, Japan), SDS (Wako Pure Chemical Industries, Ltd., Japan), and commercial grade polyoxyethylene nonylphenyl ether (Emulgen 911, Kao Co., Japan) were used as received. All water used for these experiments was purified using an ErixUV (Millipore, Japan) purification system and had a resistivity of $18.2 \mathrm{M} \Omega \mathrm{cm}$.

Preparation of PS/PMMA, PS/P(MMA-S), and PS/ P(MMA-S-tBMA) Composite Particles. PS/PMMA composite particles were prepared by seeded dispersion polymerization of MMA using PS seed particles. MMA (0.16 g), V-65 (4 mg), and PVP (K-30, $0.032 \mathrm{~g}$ ) were added to a PS dispersion [ 5 wt \% solids in methanol/water $(1.92 \mathrm{~g} / 1.28 \mathrm{~g})$ ]. The polymerization was performed at $50{ }^{\circ} \mathrm{C}$ for $24 \mathrm{~h}$ with shaking at $50 \mathrm{cycles} / \mathrm{min}$ ( $3 \mathrm{~cm}$ strokes). After polymerization, the obtained particles were washed with methanol/water via centrifugation to remove excess PVP; then, the medium was replaced with water. PS/P(MMA-S) and PS/P(MMA-S$t \mathrm{BMA})$ particles were also prepared by seeded dispersion polymerization under the same conditions.

Preparation of PS/PMMA Janus Particles. The PS/ PMMA Janus particles were prepared using the SARM process, which was proposed by Okubo et al. ${ }^{24}$ for adjusting the morphology of composite particles to obtain a thermodynamically stable structure. A typical procedure was performed as follows. Toluene $(1.2 \mathrm{~g})$, which is a good solvent for PS and PMMA, and SDS $(0.047 \mathrm{~g})$ were added to the composite particle dispersion ( $0.7 \mathrm{wt} \%$ solid content) in a $50 \mathrm{~mL}$ glass vial, and the mixture was gently stirred for several hours to absorb toluene into the composite particles. The absorbing toluene was evaporated from the dispersion by stirring with a magnetic stirrer at room temperature for $24 \mathrm{~h}$ in an uncovered glass cylindrical vessel (solvent-releasing process). After the releasing process, the SDS present on the surface of the obtained particles was removed by centrifugal washing three times with water.

Preparation of Cylindrical Janus Particles. Janus particles prepared by the SARM were deformed into a cylindrical shape via the stirring method. The particle dispersion (2.0 g, $2.0 \mathrm{wt} \%$ solids) was added to a PVP (K$15)$ aqueous solution $(2.0 \mathrm{~g}, 2.0 \mathrm{wt} \%)$ and stirred in a $15-\mathrm{mL}$ glass vial for $72 \mathrm{~h}$ using a magnetic stirrer $(360 \mathrm{rpm})$ at room temperature. A cylinder type stirring bar $(\varphi 5 \times 15 \mathrm{~mm}$, polytetrafluoroethylene) was adopted. After stirring, the particles were washed with water three times to remove excess PVP.

Hydrolysis of PS/P(MMA-S-tBMA) Cylindrical Janus Particles. To prepare amphiphilic cylindrical Janus particles, $t$ BMA units of the PMMA phase were hydrolyzed using a $6 \mathrm{wt}$ $\%$ TFA aqueous solution in a hermetically sealed glass reactor at $60{ }^{\circ} \mathrm{C}$ for $9 \mathrm{~h}$.
Preparation of the Pickering Emulsion. The Pickering emulsion was prepared by emulsifying octane (oil) and an aqueous PS/P(MMA-S- $t$ BMA) cylindrical Janus particle dispersion using a homogenizer at $6000 \mathrm{rpm}$ for $5 \mathrm{~min}$, wherein the ratio of particles/octane/water was $0.025 / 0.25$ / $1.75(\mathrm{w} / \mathrm{w} / \mathrm{w})$.

Characterization. The obtained particles were observed using an optical microscope (ECLIPSE 80i, Nikon) and a scanning electron microscope (JSM-6510, JEOL, Tokyo, Japan) at $20 \mathrm{kV}$. The number-average diameter $\left(D_{\mathrm{n}}\right)$ and coefficient of variation $\left(C_{v}\right)$ were determined by counting over 200 particles in the SEM images using image analysis software (WinROOF, Mitani Co., Ltd., Japan). To observe the interior morphology of the core-shell and Janus particles, the dried particles were stained with ruthenium tetraoxide $\left(\mathrm{RuO}_{4}\right)$ vapor at room temperature for $30 \mathrm{~min}$ in the presence of $\mathrm{RuO}_{4}$ crystal, which was embedded in an epoxy matrix, cured at room temperature overnight, and subsequently microtomed. Ultrathin (100-nm-thick) cross sections were observed using a transmission electron microscope (JEOL JEM-1230) at 100 $\mathrm{kV}$.

\section{ASSOCIATED CONTENT}

\section{Supporting Information}

The Supporting Information is available free of charge at https://pubs.acs.org/doi/10.1021/acsomega.0c04538.

Optical micrographs of PS and PMMA particles after stirring and statistics on the number of deformations, aspect ratio statistics for PS/P(MMA-S) cylindrical particles, and optical micrograph and SEM image of the PS/P(MMA-S-tBMA) (PDF)

\section{AUTHOR INFORMATION}

\section{Corresponding Author}

Hideto Minami - Department of Chemical Science and Engineering, Graduate School of Engineering, Kobe University, Kobe 657-8501, Japan; 주잉.org/0000-00016173-6597; Phone: (+81)-78-803-6197;

Email: minamihi@kobe-u.ac.jp

\section{Authors}

Miku Onishi - Department of Chemical Science and Engineering, Graduate School of Engineering, Kobe University, Kobe 657-8501, Japan

Yuya Tsujishita - Department of Chemical Science and Engineering, Graduate School of Engineering, Kobe University, Kobe 657-8501, Japan

Wei Li - Department of Chemical Science and Engineering, Graduate School of Engineering, Kobe University, Kobe 6578501, Japan

Toyoko Suzuki - Department of Chemical Science and Engineering, Graduate School of Engineering, Kobe University, Kobe 657-8501, Japan

Complete contact information is available at:

https://pubs.acs.org/10.1021/acsomega.0c04538

\section{Notes}

The authors declare no competing financial interest. 


\section{REFERENCES}

(1) Kim, J.-W.; Larsen, R. J.; Weitz, D. A. Synthesis of nonspherical colloidal particles with anisotropic properties. J. Am. Chem. Soc. 2006, 128, 14374-14377.

(2) Hosein, I. D.; Liddell, C. M. Convectively assembled nonspherical mushroom cap-based colloidal crystals. Langmuir 2007, 23, 8810-8814.

(3) Li, W.; Suzuki, T.; Minami, H. A Facile Method for Preparation of Polymer Particles Having a "Cylindrical" Shape. Angew. Chem., Int. Ed. 2018, 57, 9936-9940.

(4) Wang, Y.; Zhai, S.; Sun, H.; Zou, H. Preparation of cylinder-like polystyrene-silica composite particles. Polymer 2020, 211, 123094123102.

(5) Pang, X.; Wan, C.; Wang, M.; Lin, Z. Strictly Biphasic Soft and Hard Janus Structures: Synthesis, Properties, and Applications. Angew. Chem., Int. Ed. 2014, 53, 5524-5538.

(6) Hong, L.; Jiang, S.; Granick, S. Simple method to produce Janus colloidal particles in large quantity. Langmuir 2006, 22, 9495-9499.

(7) Suzuki, D.; Tsuji, S.; Kawaguchi, H. Janus microgels prepared by surfactant-free pickering emulsion-based modification and their selfassembly. J. Am. Chem. Soc. 2007, 129, 8088-8089.

(8) Fujimoto, K.; Nakahama, K.; Shidara, M.; Kawaguchi, H. Preparation of unsymmetrical microspheres at the interfaces. Langmuir 1999, 15, 4630-4635.

(9) Gangwal, S.; Cayre, O. J.; Velev, O. D. Dielectrophoretic Assembly of Metallodielectric Janus Particles in AC Electric Fields. Langmuir 2008, 24, 13312-13320.

(10) Okubo, M. Control of Particle Morphology in Emulsion Polymerization. Makromolekulare Chemie-Macromolecular Symposia, 1990, Vol. 35-36, pp 307-325.

(11) Nie, Z.; Li, W.; Seo, M.; Xu, S.; Kumacheva, E. Janus and ternary particles generated by microfluidic synthesis: Design, synthesis, and self-assembly. J. Am. Chem. Soc. 2006, 128, 9408-9412.

(12) Yang, S.; Guo, F.; Kiraly, B.; Mao, X.; Lu, M.; Leong, K. W.; Huang, T. J. Microfluidic synthesis of multifunctional Janus particles for biomedical applications. Lab Chip 2012, 12, 2097-2102.

(13) Dendukuri, D.; Pregibon, D. C.; Collins, J.; Hatton, T. A.; Doyle, P. S. Continuous-flow lithography for high-throughput microparticle synthesis. Nat. Mater. 2006, 5, 365-369.

(14) Liu, Y. F.; Abetz, V.; Müller, A. H. E. Janus cylinders. Macromolecules 2003, 36, 7894-7898.

(15) Tu, F.; Lee, D. Shape-Changing and Amphiphilicity-Reversing Janus Particles with pH-Responsive Surfactant Properties. J. Am. Chem. Soc. 2014, 136, 9999-10006.

(16) Yan, J.; Bloom, M.; Bae, S. C.; Luijten, E.; Granick, S. Linking synchronization to self-assembly using magnetic Janus colloids. Nature 2012, 491, 578-581.

(17) Kang, C.; Honciuc, A. Influence of Geometries on the Assembly of Snowman-Shaped Janus Nanoparticles. ACS Nano 2018, $12,3741-3750$

(18) Onishi, S.; Tokuda, M.; Suzuki, T.; Minami, H. Preparation of Janus Particles with Different Stabilizers and Formation of OneDimensional Particle Arrays. Langmuir 2015, 31, 674-678.

(19) Walther, A.; Müller, A. H. E. Janus Particles: Synthesis, SelfAssembly, Physical Properties, and Applications. Chem. Rev. 2013, $113,5194-5261$.

(20) Chaudhary, K.; Chen, Q.; Juárez, J. J.; Granick, S.; Lewis, J. A. Janus Colloidal Matchsticks. J. Am. Chem. Soc. 2012, 134, 1290112903.

(21) Kim, J.; Choi, C.-H.; Yeom, S.-J.; Eom, N.; Kang, K.-K.; Lee, C.-S. Directed Assembly of Janus Cylinders by Controlling the Solvent Polarity. Langmuir 2017, 33, 7503-7511.

(22) Saito, N.; Nakatsuru, R.; Kagari, Y.; Okubo, M. Formation of "Snowmanlike" Polystyrene/Poly(methyl methacrylate)/Toluene Droplets Dispersed in an Aqueous Solution of a Nonionic Surfactant at Thermodynamic Equilibrium. Langmuir 2007, 23, 11506-11512.

(23) Li, W.; Suzuki, T.; Minami, H. The interface adsorption behavior in a Pickering emulsion stabilized by cylindrical polystyrene particles. J. Colloid Interface Sci. 2019, 552, 230-235.
(24) Saito, N.; Kagari, Y.; Okubo, M. Effect of Colloidal Stabilizer on the Shape of Polystyrene/Poly(methyl methacrylate) Composite Particles Prepared in Aqueous Medium by the Solvent Evaporation Method. Langmuir 2006, 22, 9397-9402. 